\title{
MODELISATION DES MICROPHONES MINIATURES : EFFETS DES COUCHES LIMITES VISCOTHERMIQUES
}

\author{
M. BRUNEAU, A.M. BRUNEAU et P. HAMERY \\ Laboratoire d'Acoustique, Associé au C.N.R.S. (U.R.A. 1101), Université du Maine, Avenue Olivier \\ Messiaen, BP. 535, F-72017 Le Mans cedex, France
}

\begin{abstract}
The purpose of this paper is to calculate the acoustic response of a stretched membrane separated from a rigid electrode by an air film whose thickness is the same order of magnitude as the viscous and thermal boundary layers(in the range of 1-10 $\mu \mathrm{m})$, tacking into account the effect of a very small annular volume. The goal is to obtain an approximate model to describe the behavior of miniaturised condenser microphones designed on a silicon substrate, from the basic equations involving viscous, thermal, and acoustic effects.
\end{abstract}

\section{1- Introduction.}

Le développement actuel des transducteurs miniatures sur puces en silicium doit conduire, dans les années prochaines, à l'arrivée de nouveaux produits sur le marché $[1,2]$. Il en est ainsi en particulier des microphones à condensateurs miniatures qui ont fait l'objet d'études et de développements ces dernières années. L'objectif visé à l'heure actuelle concerne principalement le marché de la prise de son usuelle; mais cette technologie sur silicium autorise des applications diverses dans le domaine sonore (embouts d'oreilles, casques d'écoutes, capteurs à directivité controlée,...) comme dans celui du proche ultrasonore (gyromètre miniature, "scale modelling",...).

La modélisation de ces transducteurs microphoniques [3], telle qu'elle apparaît aujourd'hui, n'est qu' une transposition de celle en usage dans les microphones classiques, alors que les espaces clos inclus dans les puces silicium ont des dimensions du même ordre de grandeur que celles des couches limites visqueuses et thermiques d'origine acoustique, ce qui confère aux phénomènes tourbillonnaires et entropiques une importance "inhabituelle".

Parce qu'il semble aujourd'hui utile de proposer des modèles de nature à permettre de maîtriser l'étude prévisionnelle de ces transducteurs (utilisables aussi bien en émetteurs qu'en récepteurs), mais aussi parce que de diverses manières, apparaît le besoin de modéliser les champs en cavités de plus en plus petites avec une précision accrue, il est nécessaire de nos jours d'approfondir les études de champs acoustiques en espace clos en prenant en compte leurs interactions fortes avec les mouvements tourbillonnaires et les mouvements entropiques (liés au flux de chaleur).

C'est dans ce cadre qu'entre le travail présenté ici, qui repose sur des études récentes dont l'objet était d'une part d'adapter au calcul des champs en cavités miniatures les connaissances de problèmes de propagation en fluide viscothermique [4,5], et d'autre part de montrer, par une étude 
comparative, l'importance des mouvements entropiques négligés jusqu'alors dans le calcul des transducteurs capacitifs [6].

\section{2- Le problème.}

Les équations du problème sont celles qui régissent la propagation en fluide viscothermique. Ce fluide oscille autour de son état d'équilibre qui peut être décrit par un ensemble de paramètres, dont la valeur ambiante $\rho$ de la masse volumique et par les coefficients en usage dans la description du comportement des fluides (coefficients de viscosité $\mu$ et $\eta$, de conduction thermique $\lambda$, capacités calorifiques $C_{p}$ et $C_{v}$ et leur rapport $\gamma$ ) et par un ensemble de variables dynamiques dont la pression acoustique $p$, la vitesse particulaire $v$ ( somme d'une vitesse laminaire $v_{\ell}$ et d'une vitesse tourbillonnaire $v_{v}$, la variation de la masse volumique $\rho$, la variation d'entropie $s$ et la variation de température $\tau$, toutes étant supposées petites de façon à rester dans le domaine de l'acoustique linéaire.

Les lois fondamentales du mouvement s'expriment, sous leur forme linéarisée, par :

- L'équation de Stokes-Navier

$$
\rho a_{t} v+\operatorname{grad} p=\left(\frac{4}{3} \mu+\eta\right) \operatorname{grad} \operatorname{div} v-\mu \operatorname{rot} \operatorname{rot} v
$$

où c désigne la vitesse du son.

- L'équation de la conservation de la masse, compte tenu du fait que l'écart instantané de masse volumique est une différentielle totale,

$$
\rho c^{2} \operatorname{div} v+\gamma \partial_{t}(p-\beta \tau)=0
$$

- L'équation de Fourier pour la conduction de la chaleur, compte tenu du fait que l'entropie est une fonction d'état,

$$
\left.\left[\frac{1}{c} \partial_{t}-\frac{\lambda}{\rho c C_{p}} \Delta\right] \tau=\frac{\gamma-1}{\beta \gamma C} \partial_{t} p \quad, \beta=\frac{\partial P}{\partial T}\right)_{\rho}
$$

La vitesse particulaire se décompose en une vitesse tourbillonnaire vérifiant les équations $\left(\rho \partial_{t}-\mu \Delta\right) v_{v}=0$ et $\operatorname{div} v_{v}=0$, et une vitesse laminaire exprimée par $v_{\ell}=-\operatorname{grad} \varphi$ où $\varphi$ est le potentiel des vitesses en fonction duquel la pression acoustique $p$ et la variation de température $\tau$ sécrivent :

$$
\begin{aligned}
& p=\rho \partial_{t} \varphi-\left(\frac{4}{3} \mu+\eta\right) \Delta \varphi \\
& \tau=\beta^{-1}\left[\rho \partial_{t} \varphi-\left[\frac{\rho c^{2}}{\gamma} \partial_{t}^{-1}+\left(\frac{4}{3} \mu+\eta\right)\right] \Delta \varphi\right] .
\end{aligned}
$$

Dans le système microphonique étudié, le fluide considéré est confiné dans l'espace qui sépare deux électrodes, l'une une membrane en $z=d$, $l^{\prime}$ autre une électrode rigide fixe en $z=0$ (d de 1 'ordre de 1 à $10 \mu \mathrm{m}$ ), fermé latéralement par une petite cavité périphérique (reliée en pratique à l'extérieur par un tube capillaire d'égalisation de pression). 


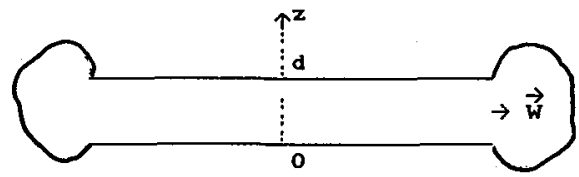

Microphone à condensateur : notations.

Les conditions aux limites du problème sont les suivantes :

- Variation de température nulle sur les électrodes

$$
\tau=0 \quad \text { en } z=0 \text { et en } z=d
$$

- Vitesse particulaire tangentielle nulle sur les électrodes

$$
\mathbf{n}_{w} \cdot\left(\operatorname{grad} \varphi+v_{v}\right)=0 \quad \text { en } z=0 \text { et en } z=d
$$

- Vitesse particulaire normale nulle sur l'électrode fixe

$$
\mathbf{n}_{\mathbf{z}} \cdot\left(\operatorname{grad} \varphi+\mathbf{v}_{\mathbf{v}}\right)=0 \quad \text { en } \quad z=0
$$

- Continuité de la vitesse particulaire normale sur la membrane

$$
\mathbf{n}_{\mathbf{z}} \cdot\left(\operatorname{grad} \varphi+\mathbf{v}_{\mathbf{v}}\right)=\partial_{t} \xi \quad \text { en } \quad z=d \text {, }
$$

où $\xi$ désigne le déplacement de la membrane (suivant l'axe $z$ ).

- Condition de continuité à l'interface entre lame d'air et volume périphérique, choisie de type impédance.

\section{3 - Les solutions.}

Sous l'effet de la pression extérieure à mesurer, la membrane entre en vibration. Ce mouvement peut être décrit par un développement sur la base des fonctions propres de la membrane, écrites, pour les besoins des calculs, comme la somme d'une onde divergente, et d'une onde convergente, ou par une solution à caractère explicite.

Af in d'obtenir une expression analytique approchée de nature à donner les termes à introduire dans les modèles électriques équivalents en usage dans les descriptions classiques des transducteurs capacitifs, la solution pour le potentiel des vitesses du gaz confiné dans l'entrefer est cherchée de manière à faire apparaître un facteur compatible avec le mouvement de la membrane, associé aux modes propres de la lame d'air, reliés entre eux par la condition de réflexion à $l^{\prime}$ interface entre la lame d'air et le volume périphérique.

\section{4 - Les résultats actuels.}

La recherche de ces modèles qui doivent tenir compte de ce que l'espace dans l'entrefer est du même ordre de grandeur que l'épaisseur des couches limites (quelques micrométres) est actuellement en cours, mais les prototypes, calculés au moyen des procédés disponibles [7], ont été réalisés. L'insuffisance actuelle de leurs caractéristiques en regard des exigences liées aux usages qui leurs sont destinés confirme la nécessité des études approfondies entreprises.Les premiers prototypes réalisés par 1'E.S.I.E.E. [8] et en collaboration avec la société SEXTANT AVIONIQUE, présentent une courbe de sensibilité en fonction de la fréquence dont l'allure est présentée sur le diagramme qui suit. 


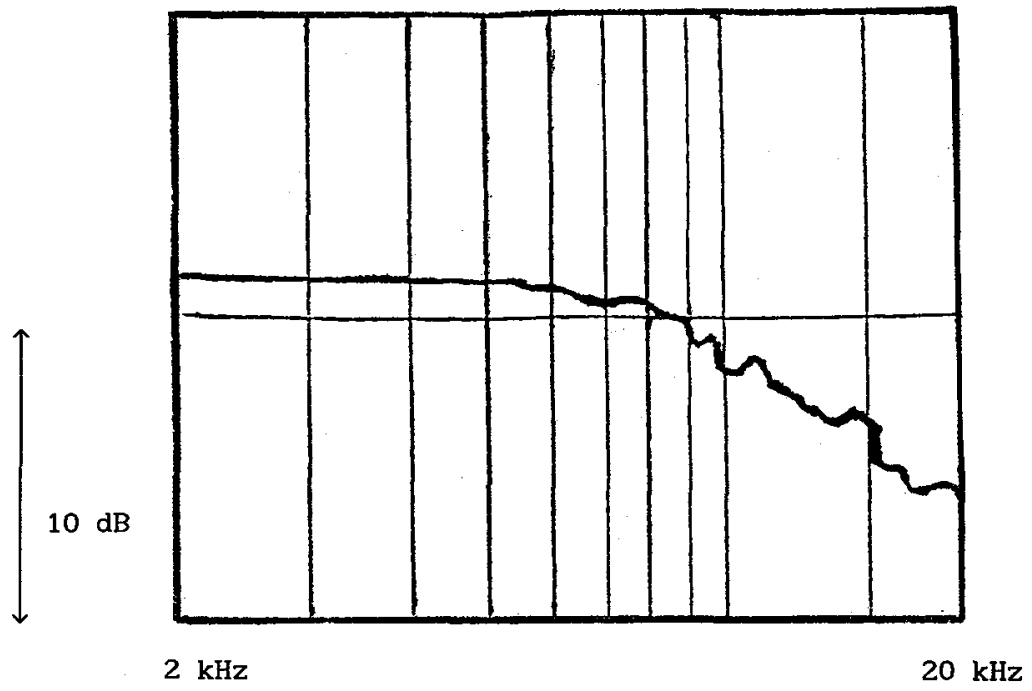

Sensibilité en fonction de la fréquence d'un microphone miniature (fabrication E.S.I.E.E. sur silicium). $\begin{array}{ll}\text { Surface de la membrane } & 1 \mathrm{~mm}^{2} \\ \text { Epaisseur de la lame d'air }: 7.510^{-6} \mathrm{~m} \\ \text { Sensibilité nominale } & : 1 \mathrm{mV} / \mathrm{Pa} .\end{array}$

5 - Conclusion.

Pour diverses applications, la fréquence de coupure haute dolt être beaucoup plus élevée que celle obtenue sur ces premiers prototypes.Pour cette raison et pour d'autres liées à la sensibilité, à la réponse en phase, etc ...l, obtention de modèles plus précis que ceux disponibles à l'heure actuelle est rendue nécessaire.

\section{Références :}

[1] Hohm D., Sessler G.M., "An integrated silicon electret condenser microphone ", Proceedings of the International Congress on Acoustics, Paris, 1983, Rev. Acoust. 6, (1983) 29-32.

[2] Hohm D., Hess G., "A subminiature condenser microphone with silicon nitride membrane and silicon back plate", J.Acoust.Soc. Am. 85 (1989) 476-480.

[3] Zdeněk Skvor, "Vibrating systems and their Equivalent Circuits", Elsevier, 1991.

[4] M. Bruneau, P.Herzog, J.Kergomard, J.D.Polack, "General formulation of the dispersion equation in bounded viscothermal fluid, and application to simple geometries", Wave Motion 11 (1989) 441-451.

[5] J.D.Polack, "Time domaine solution of Kirchoff's equation for sound propagation in viscothermal gases : a diffusion process", J.Acoustique 4 (1991) 47-67.

[6] G.Plantier, M. Bruneau, "Heat conduction effects on the acoustic response of a membrane separated by a very thin air film from a backing electrode". J.Acoustique 3 (1990) 243-250.

[7] Zdenèk Skvor, "Transducteurs electroacoustiques et electrodynamiques et leurs circuits equivalents", $2^{\text {eme }}$ C.F.A., Arcachon, 1992.

[8] T. Bourouina, "Conception, réalisation et caractérisation d'un microphone miniaturisé en technologie silicium", Thèse de Doctorat. Paris XII - Val de Marne. 